\title{
Research on Platform Lighting Control System Based on Wireless Sensor Network
}

\author{
HAN Ke ${ }^{1, a,}$, ZHAO Sihai ${ }^{1, b^{*}}$, HU Yunlan ${ }^{1, c}$,WANG Xiaoshu ${ }^{2, \mathrm{~d}}$,XU Jiangye ,e $^{1, \mathrm{e}}$ \\ ${ }^{1}$ School of Mechanical Electronic and Information Engineering,China University of Mining and \\ Technology,Xueyuan Road, Beijing,China \\ ${ }^{2}$ Institute of Computing Technology, China Academy of Railway Sciences,DaLiuShu Road, Beijing,China \\ ahanke_122@163.com, bzsh@cumtb.edu.cn, c15801388711@163.com,

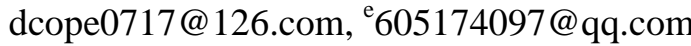

Keywords: lighting control, industrial PC, configuration software, wireless sensor network, illuminance

\begin{abstract}
In view of the backward lighting control methods in many domestic railway stations,an automatic lighting control scheme using industrial PC as control core and wireless sensor network to collect illuminance information for feedback control is proposed.The wireless sensor network is designed to achieve real-time collection and wireless transmission of illuminance data.Besides,a intelligent lighting controller based on fuzzy control algorithm is invented independently to achieve the closed-loop feedback of lighting control system.Finally, using Force Control configuration software to achieve the entire control system software design, and commissioning test.The system has been applied in Taiyuan station. It achieved the remote and automatic control on platform lighting, and solve the problem of waste of power resources effectively.
\end{abstract}

Chinese railway transportation industry is developed and the speed of development is accelerating. At present, there are about 5544 railway stations in China, which play an important role in the transportation industry. Although the development of railway transportation in China is rapid in recent years, the basic construction of the station lighting controlling system is relatively backward, especially in some existing stations. To improve the quality of passenger service, the modern, reliable, intelligent and efficient management requirement has been raised in controlling requirements of the lighting controlling system of the railway station by China Railway General Corporation $^{[1]}$. The number of electric light in railway station platform is numerous and the demand of electric energy is huge. For example, there are 1319 gold halogen electric light in the 1-7 platform of Taiyuan Railway Station and the annual electricity consumption is $1011014 \mathrm{kWh}$. Because of relatively backward management controlling system, the automation construction is insufficient, so that the switches of the platform electric light need frequent manual operation of the staff, which brings inconvenience to them. And due to the ignorance of the staff, the lighting fixtures of the platform are easy to appear in the daytime. This manual controlling method is backward and tedious, and numerous waste of resources and low efficiency are caused. Lighting system has been an important part of the railway station. Therefore, to reduce the waste of energy and achieve the goal of energy saving, the design of lighting controlling system has become an important content of the construction of modern railway station ${ }^{[2]}$.

Nowadays, to achieve intelligent controlling of railway lighting, more and more resources are input in the design of intelligent lighting controlling systems all over the world ${ }^{[3]}$. With the development of intelligentization, the requirement of lighting controlling system for railway

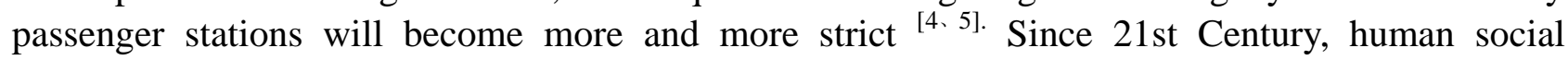
civilization has made great progress because of revolutionary historical significance the development of wireless sensor networks brought ${ }^{[6]}$. The wireless sensor network and fuzzy controlling are introduced into the research of the lighting system respectively. Both of them provide thoughts and application prospects for the development of intelligent lighting ${ }^{[7 \sim 9]}$. Wireless sensor network is composed of a large number of sensor nodes with low power consumption, high 
degree of integration and low cost ${ }^{[10]}$. And its ability includes collecting information, processing data and wireless communication, and so on ${ }^{[11]}$. If the automatic and intelligent controlling of railway station lighting based on wireless sensor network can be realized, and the lighting fixtures of the platform can be automatically controlled according to the light conditions of the outside conditions, it can save a lot of energy, reduce the number of electric light, avoid unnecessary energy waste and reduce the pressure of staff and items At the same time the system achieve intelligent controlling requirements, and improve the comprehensive management ability and work efficiency of the platform.

Based on the conditions of low efficiency of lighting controlling and waste of resources, the platform lighting controlling system is upgraded accordingly. A new platform lighting controlling system is developed by the usage of wireless sensor, WIFI and ethernet, which organize a distributed network in the network system to achieve the automation and intelligent function of the controlling of the lighting fixtures of the platform and controlling switches of the electric light reasonably. On the one hand, it can provide people with comfortable and harmonious lighting environment. On the other hand, it can protect the electric light, extend the service life, save the cost, and meet the requirements of green environmental protection.

\section{The Design of Lighting Controlling System}

\subsection{The Principle of Controlling}

The principle of negative feedback controlling theory is mainly applied by the platform intelligent lighting controlling system. Firstly, the actual illumination intensity of the platform is collected by each platform illuminance sensor, and it will be compared with the settings to feedback, which is settled by the staff according to the demand. Secondly, after comparison the light intensity results data is transferred to the digital controller, and the controlling signal is sent out by the digital controller to act on the intersection of the light intensity. Finally, closed-loop controlling is achieved and the system is automatic controlling by controlling the AC contactor which is used for the opening and closing of the electric light. The controlling schematic diagram of the whole system is shown in the following figure:

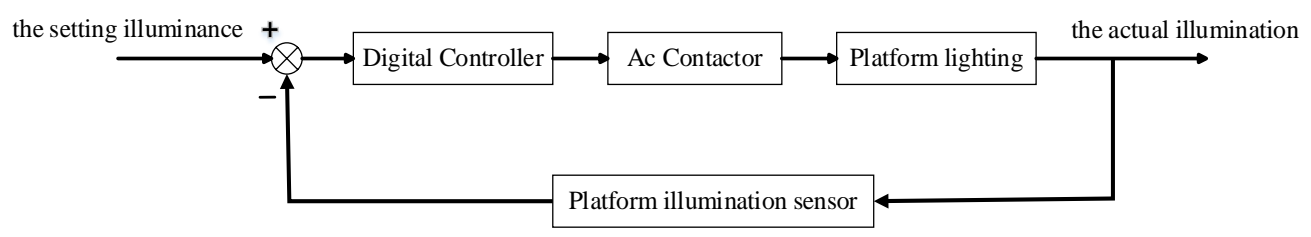

Figure 1 System control schematic diagram.

\subsection{The Structure of System}

The controlling core of the system is an industrial PC. Firstly, the measured data collected by illuminance sensor is sent to the upper computer through the wireless transmission of the WIFI, and the control signal is sent out to the platform intelligent controller after received data disposed by the upper computer. Secondly, the control signal is received by the intelligent controller from the host computer through the wired Ethernet, and the contactor function as the switch of the electric light according to the output control signal after the control signal processed by the program. The platform intelligent lighting controlling system is connected with each platform lighting controller through switches, and the electric light of this platform is controlled by each platform lighting controller. And each platform lighting controller is composed of industrial microcomputer, data acquisition card and contactor. The system topology is shown as shown in the following figure. 


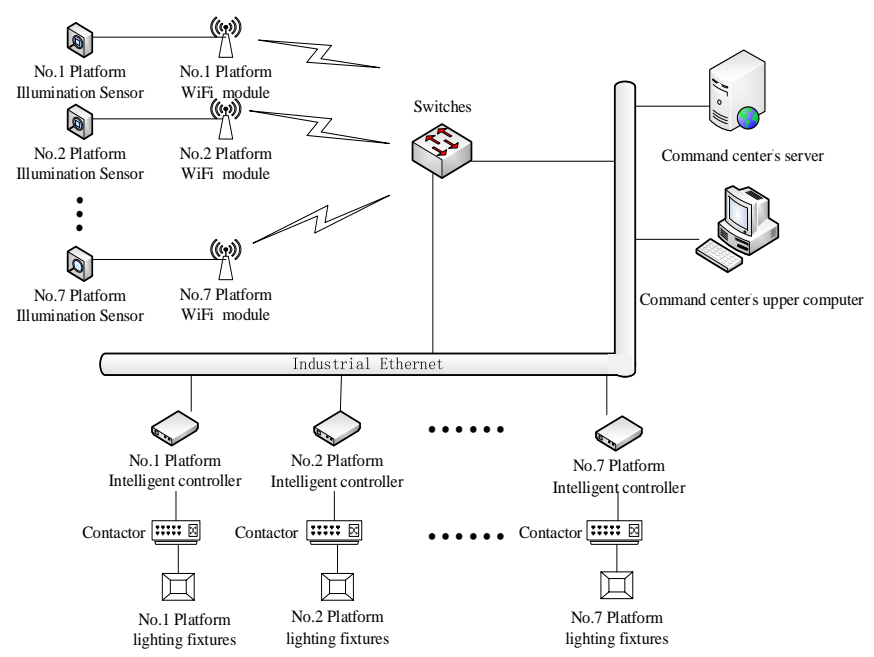

Figure 2 Network topology of lighting control system.

\section{Hardware Design of The System}

\subsection{Hardware Design of Sensor Subsystem}

The illuminance wireless sensor in the lighting control system is installed behind the platform LED panel generally. On the one hand ,the collection of optical data is not affected; on the other hand, it can achieve beautiful installation effect.

The sensor subsystem is mainly composed of a SCM, a luminance sensor and a wireless WiFi module, and it is in charge of collecting and transmitting illuminance information ${ }^{[12]}$.STC15W4K56S4 is used into the design. Using GY-30 as the illuminance detection module, BH1750FVI as a chip. The wireless WiFi module use ESP8266, which is connected with STC15 through serial port.In the state of normal work,, the sensor subsystem collects illuminance data by GY-30 illuminance detection module, the data is sent to the wireless AP through the ESP8266 wireless WiFi module after processed by STC15and accessed to the station Ethernet.Then the data can be transferred to the upper computer in the control room to realize long-distance wireless transmission.

\subsection{Hardware Design of The Intelligent Controller}

The intelligent controller is mainly composed of a master controller, a USB data acquisition card and a relay module.Based on the current situation of the small platform control cabinet, the Colored GlazeTM mini computer host is selected as the mini PC .The computer host has the USB2.0 interface, which can be connected with the relay board by USB data acquisition card to output the control signal.

Adding MC100 USB data acquisition card between relay board and PC.The controlling software in mini PC output control signal.Firstly,the signal is transmitted to the MC100 data acquisition card through the USB port; then,the MC100 output digital quantity to control the relay module;finally, through controlling the pick-up of contactor which is controlled by relay to control the platform of lighting.

\section{Software Design of The System}

The software design of intelligent control system mainly includes: software design of sensor subsystem and software design of intelligent controller.

In this paper,the whole lighting control system is completed under the platform of the force control configuration software.I/O driver management, real-time database system, monitoring screen and WEB publishing constitute the software architecture of the system. The real-time data of platform sensor equipment is collected by real-time database,and on the one hand,it goes to the intelligent controller through I/O data connection after processed by the configuration software;on 
the other hand,through the management of the monitoring software,it will be displayed on the monitor screen and published on the WEB.Configuration software structure of the system is shown in Figure 3.

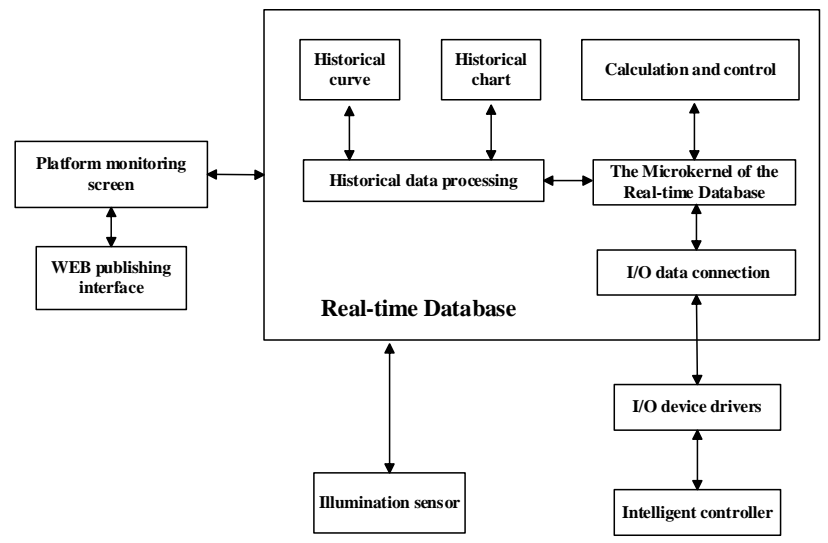

Figure 3 Configuration software structure diagram.

\subsection{Software Design of Sensor Subsystem}

The data collection of illumination module is realized by BH1750FVI chip.And the collected data is sent wirelessly by ESP8266 WiFi wireless module after scaling,digital filtering and other processing.Sensor subsystem control flow chart is shown in Figure 4.

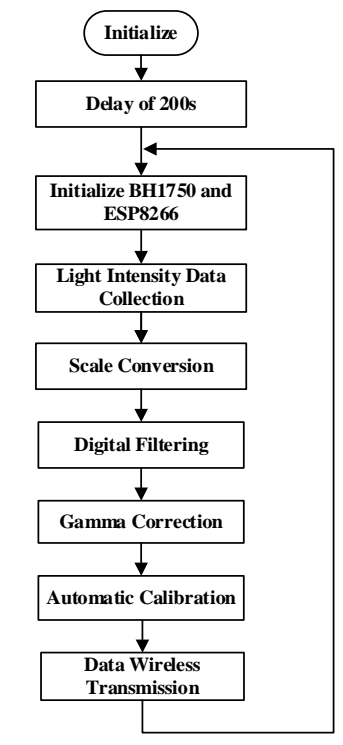

Figure 4 Sensor subsystem control flow chart.

\subsection{Software Design of Intelligent Controller}

\subsubsection{The Design of Control Algorithm}

In this paper,the writers adopt 2D fuzzy control ${ }^{[13,14]}$. Based on the general method of fuzzy control, the fuzzy variable are:e( the illuminance value),ec( the gradient of illuminance value), $u($ the output).The input and output variables can be expressed as:negative large(NB),negative little(NS),negative zero(NZ),positive zero(PZ),positive large(PB),positive little(PS).The basic domain of illuminance value and gradient of illuminance value are $+\mathrm{e}$ 、 + ec respectively.Their range are $[-5,+5]$ and the accurate quantity can be divided into 12 levels. The domain and range of output are $+\mathrm{u}$ and $[-6,+6]$, and it's accurate quantity can be divided into different grades.The system has two inputs and one output,according to the previous experience of control, the reasoning language rules as shown below:

$$
\text { if } \mathrm{E}=(\mathrm{NB}) \text { and } \mathrm{EC}=(\mathrm{PB})
$$




$$
\text { then } \mathrm{U}=(\mathrm{PB})
$$

For example,if the illuminance value $\left(E=e^{\prime}\right)$ as the negative large and the gradient of illuminance value $\left(\mathrm{EC}=\mathrm{ec}^{\prime}\right.$ ) as the positive large,the output control increment $(\mathrm{U}=\Delta \mathrm{u})$ should be positive large to reduce the negative deviation and make it close to the given value.By reasoning fuzzy condition statements, the following fuzzy relation can be determined as below:

$$
\mathrm{R}=\mathrm{U}(\mathrm{E} \times \mathrm{C}) \mathrm{U}
$$

In the formula, $U$ and $X$ is equal to the " union" and "intersection" of the fuzzy relation matrix.The incremental fuzzy set $U$ is obtained by E, EC and the above inference synthesis rules.

$$
\mathrm{U}=(\mathrm{E} \times \mathrm{EC}) \mathrm{R}
$$

After the control decision $U$ is obtained, the control increment $\Delta \mathrm{u}$ which has been obtained according to the median of the membership function and the fuzzy control table which is obtained through the debugging and correction of the computer ${ }^{[15]}$ can be obtained.When in real-time processing of data, a control quantum is obtained by looking up the fuzzy control table which has been obtained according to the fuzzy value of the input deviation and the input deviation rate.Fuzzy control flow chart is shown in Figure 5.

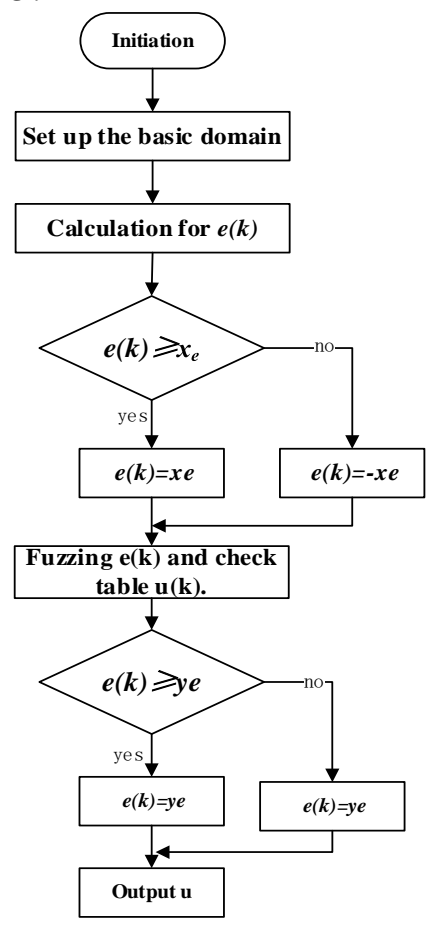

Figure 5 Fuzzy control algorithm flow chart.

\subsubsection{Communication between $P C$ and data acquisition card MC100}

The communication between PC and data acquisition card is realized by force control configuration software.Firstly,setting up the I/O device configuration in the configuration software,and selecting DDE communication mode,then configuring the device;secondly,makeing the corresponding I/O port of the data acquisition card MC 100 output low level;finally through controlling the pick-up of relay to control lighting.Flow chart of force control main program is shown in Figure 6. 


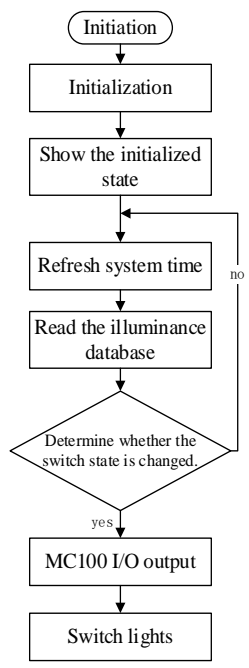

Figure 6 Flow chart of force control main program.

\section{Application}

The collection of illuminance data is an important part of the intelligent lighting control system.The following random day is taken as an example to collect the Illuminance data of the platform and collection time was from 0:30am to 11:30pm,Aug.25,2017. The number of sensors collected was 5,they were placed at the station 1 to 5 of the Taiyuan railway station. Making a line chart of sensors' data of different locations. The variation trend of different sensors' illuminance can be seen from the chart.The acquisition curve of illuminance is shown below.

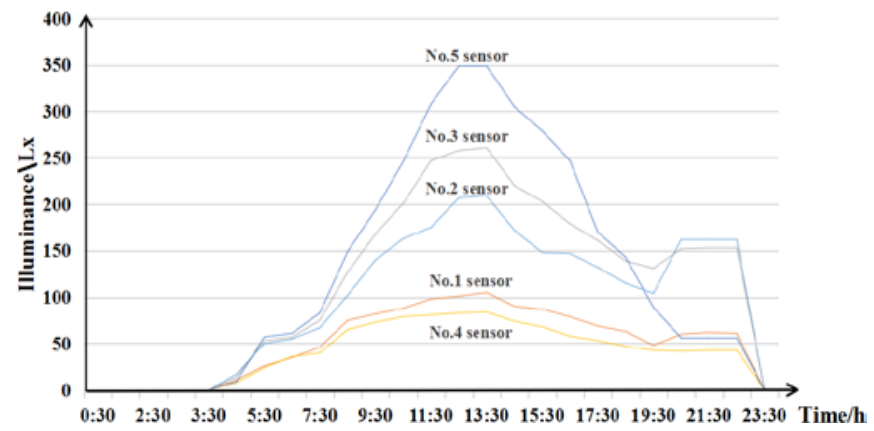

Figure 7 The light intensity data curve.

According to the change rule of illumination, the illumination threshold range of lights-out mode, half-open mode and full open mode can be delimited.

After a large number of experiments,the design of intelligent lighting control system has been put into operation in Taiyuan railway station. The system has achieved good results, met intelligent and automatic control requirements, and saved a lot of manpower and material resources. The final result of the station's intelligent lighting control system is shown in figure 8 below.

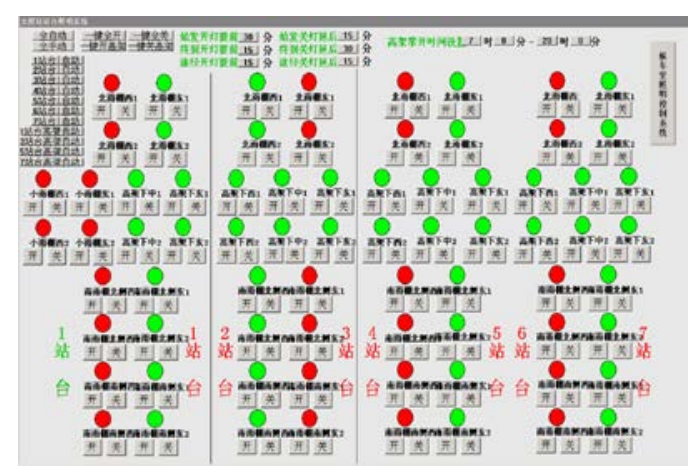

Figure 8 Operation result of upper computer control system. 


\section{Conclusion}

The lighting control system develops a new type of lighting controller based on PC - based idea , intelligent wireless sensor is applied to this control system, illumination sensor can monitor the external illumination and send data to the upper computer at the same time, the upper computer outputs signals to control platform intelligent controller through program analysis and processing,and then realize lighting auto-control.

The system has been applied in Taiyuan railway station successfully, and it has realized the automation of lighting fixtures on and off according to the external illumination condition, and the staff can monitor the running status of the lamp in real time.After applying the illumination control system, the labor cost the energy consumption is reduced, and the working efficiency is increased.It has been effective to solve the problem of wasting power resources, which is of great significance to the modern railway station with high efficiency, energy saving and intelligent environmental protection.

\section{References}

[1] Liao Mei. Lighting System Energy Saving Design on Large Railway Station [D]. (Huazhong University of Science and Technology, 2011.

[2] Zhao Yajun. Research and Design of Intelligent Lighting System Based on CAN Bus [D]. Wuhan University of Science and Technology, 2012.

[3] A.Guillemin, N.Morel.An innovative lighting controller integrated in a self-adaptive building control system.Energy and Buildings, 2001, 33(5):477 487.

[4] Wang JianTao,Leren,Zhang Zongnan. Control System Exploiting of Heat Transfer Enhancement Outside Single-tube Based on Forcecontrol [J]. Research and Exploration in Laboratory,2013,32(11):46-48+140.

[5] Meng Xiangbin, Mao Hongyan, Wang Dejun. Dedign of intelligent lighting control system.Journal of Shenyang Institute of Engineering:Natural Science,2015, 11(1) :70-73

[6] Jane K Hart, Kirk Martinez. Environmental Sensor Networks: A revolution in the earth system science7[J].Earth-Science Reviews,2006,78(3):177-191.

[7] Qin Xuping,Tan Guojun. Development and Prospect of Fuzzy Control Theory [J], Control Engineering of China ,2005,12(S2): 141 -143.

[8] G.Fraisse, J.Virgone, J.J.Roux, Thermal control of a discontinuously occupied building using a classical and a fuzzy logic approach, Energy build,1997,26 (3):303-316.

[9] Mateja Trobec Lah, Borut Zupancic, Joze Peternelj,etc. Daylight illuminance control with fuzzy logic. Solar Energy,2005,80 (3) :307 321.

[10]Chen Zongcehng. Research on Wireless Sensor Networtks Baesd on IEEE 802.15.4 [D],South China University of Technology , 2013.

[11]AiLi,Nie Danfeng. Environmental Monitoring System Based on ZigBee Wireless Technology [J],South China University of Technology ,Microprocessors, 2016,04(37):87-89+95.

[12]Dutta S, Das R, Sarkar A. Microcontroller Based Data Acquisition System[J]. International Journal of Engineering,2013, 2(7) :23-30.

[13]Wang Pengyu,Liu Wenjun. Design of Wtaer-heater Intelligent Controller Based on Solar [D]. Modern Electronics Technique,2014(5): 124-126.

[14]Guo Shenghui,Zhu Xueli,Zhu Shuxian.Research of Lighting System of Classroom Based on Fuzzy Control [J]. Zhaoming Gongcheng Xuebao, 2013,24(3):127-130.

[15]Sun Peiyan. Study and Simulation of Centrifugal Compressor Anti-Surge control Scheme Based on Fuzzy Controller[D].Harbin: Harbin Institute of Technology. 